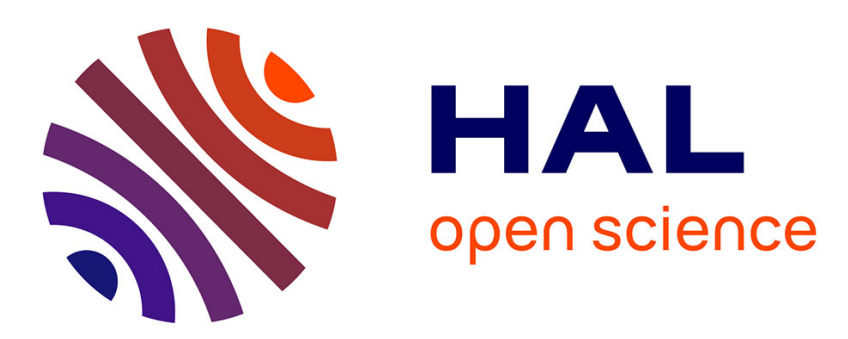

\title{
Magnéto-météorologie: discussion d'un effet atmosphérique sur le champ magnétique terrestre et de ses conséquences
}

\author{
P. Bernard
}

\section{- To cite this version:}

P. Bernard. Magnéto-météorologie: discussion d'un effet atmosphérique sur le champ magnétique terrestre et de ses conséquences. Revue de Physique Appliquée, 1970, 5 (1), pp.239-241. 10.1051/rphysap:0197000501023900 . jpa-00243368

\section{HAL Id: jpa-00243368 https://hal.science/jpa-00243368}

Submitted on 1 Jan 1970

HAL is a multi-disciplinary open access archive for the deposit and dissemination of scientific research documents, whether they are published or not. The documents may come from teaching and research institutions in France or abroad, or from public or private research centers.
L'archive ouverte pluridisciplinaire HAL, est destinée au dépôt et à la diffusion de documents scientifiques de niveau recherche, publiés ou non, émanant des établissements d'enseignement et de recherche français ou étrangers, des laboratoires publics ou privés. 


\title{
MAGNÉTO-MÉTÉOROLOGIE : DISGUSSION D’UN EFFET ATMOSPHÉRIQUE SUR LE GHAMP MAGNÉTIQUE TERRESTRE ET DE SES GONSÉQUENGES
}

\author{
Par P. BERNARD, \\ Faculté des Sciences de Paris, Institut de Physique du Globe, Paris.
}

\begin{abstract}
Résumé. - Dans un travail antérieur, nous avons trouvé l'explication de la variation undécennale de la composante horizontale du champ terrestre (dont l'amplitude est de $20 \gamma$ ) dans les vents de la couche de transition de l'ionosphère où les ions positifs sont entraînés par leurs collisions avec les atomes neutres, tandis que les électrons négatifs sont bloqués dans le sens transversal au champ terrestre. Nous montrons que le phénomène rend compte quantitativement des résultats observés, compte tenu de la variation d'ionisation à cette altitude au cours du cycle solaire. L'existence d'une variation biennale de la même composante, d'une amplitude de $5 \gamma$, s'explique par le même mécanisme en admettant une variation de vitesse des vents ionosphériques du même ordre de grandeur que celle des vents stratosphériques.

L'extrapolation de ces résultats aboutit à montrer qu'un accroissement raisonnable de l'ionisation de la haute atmosphère suffirait à expliquer un renversement du champ terrestre observé au sol, et même la naissance de ce champ à partir du faible champ interplanétaire. En conclusion, on montre l'accord de ces remarques avec les mesures magnétiques déjà obtenues au voisinage des autres corps planétaires.
\end{abstract}

Abstract. - For the explanation of the undecennal and biennal periods of $\mathrm{H}$ (horizontal geomagnetic force), ionospheric winds are considered in a transition layer (90 to $120 \mathrm{~km}$ height) where positive ions are carried along by their impacts with neutral atoms, while negative electrons are inhibited to move perpendicularly to the geomagnetic field. The wind is therefore in this case equivalent to an electric current. The actual amplitudes of the variations of $\mathrm{H}$ agree with the known data at these heights.

The formation of a transition layer in a very small magnetic field is discussed : it can give rise, for convenient velocities and directions of wind, to a much stronger field which will become permanent if it occurs during the cooling of the earth's crust. It is suggested that the magnetic fields in Sun and other celestial bodies result from a similar mechanism.

A complete English text of this paper has been distributed at the symposium, and may be obtained from the author.

Dans une précédente publication [1], nous avions étudié la variation périodique undécennale du champ géomagnétique horizontal en 37 stations à partir des séries les plus prolongées de valeurs annuelles publiées au «Thesaurus of values ». L'ensemble des résultats obtenus montrait un minimum de $\mathrm{H}$ peu après le maximum d'activité solaire ( $f i g .1$ ), et une

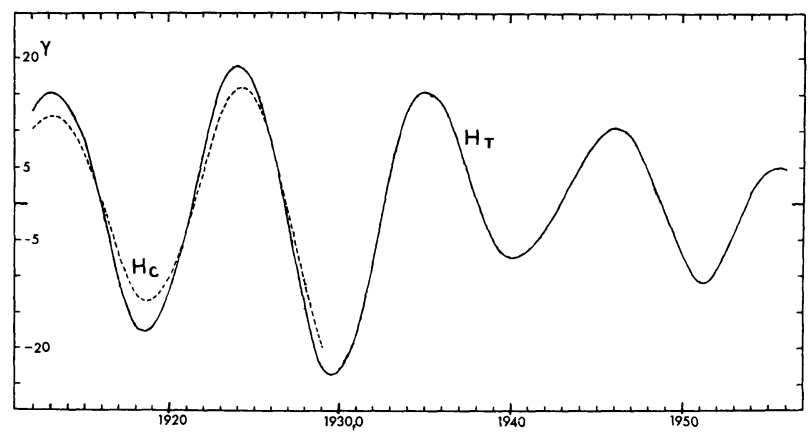

Fig. 1. - Exemple de la variation undécennale de $H$ station d'Honolulu, d'après les données du Coast and Geodetic Survey. En pointillé : courbe déduite des moyennes annuelles des jours calmes internationaux. amplitude totale crête à crête de $20 \gamma$ en moyenne; cette amplitude est très peu réduite (de $20 \%$ au plus) si, au lieu de considérer les moyennes de tous les jours, nous retenons seulement celles des jours calmes internationaux, ce qui élimine, comme cause de cette variation undécennale, l'effet de post-perturbation qui fait baisser la valeur de $\mathrm{H}$ dans les jours suivant le déclenchement des orages magnétiques.

Il existe une autre cause possible de cette période undécennale [2] : la variation du taux d'ionisation de l'atmosphère à l'altitude de transition où les vents zonaux entraînent les ions positifs, tandis que le mouvement des électrons négatifs est bloqué dans la direction perpendiculaire au champ magnétique terrestre, car leur fréquence de collision [3] est inférieure à leur fréquence gyromagnétique au-dessus de $100 \mathrm{~km}$ le jour, $90 \mathrm{~km}$ la nuit : les collisions avec les atomes neutres animés de la vitesse du vent ne font donc que relancer leur enroulement sur place autour des lignes de force du champ magnétique, tandis que les ions positifs, dont la circonférence gyromagnétique est plus longue en raison de leur masse plus élevée, ne peuvent la parcourir entièrement sans recevoir de 
nouvelles impulsions dans la direction du mouvement d'ensemble du milieu ambiant.

Ces conditions physiques interviennent à une altitude comprise entre 100 et $120 \mathrm{~km}$. Un vent zonal y donnera donc naissance à une nappe horizontale de courant dont le champ magnétique au sol :

$$
H=2 \pi i
$$

sera en sens contraire du champ terrestre pour un déplacement d' $E$ en $W$ des charges ioniques positives : la différence de $20 \gamma$ entre les extrêmes du cycle undécennal, si l'on admet une variation d'ionisation de $10^{5}$ charges élémentaires par $\mathrm{cm}^{3}$, résulterait d'un vent moyen de $100 \mathrm{~m} / \mathrm{s}$ sur une épaisseur de $20 \mathrm{~km}$. Tous ces chiffres sont d'un ordre de grandeur conforme à la réalité : on sait en particulier que les nuages lumineux nocturnes accusent des vitesses de déplacement d' $E$ en $W$ souvent supérieures à $100 \mathrm{~m} / \mathrm{s}$.

Un travail récent semble confirmer l'action atmosphérique sur le champ magnétique que nous proposons : on retrouve dans les valeurs de $H$ la variation de 2 ans découverte dans les vents stratosphériques équatoriaux. Nous avons pu la mettre nettement en évidence à Huancayo [4], station justement équatoriale par la méthode des combinaisons linéaires d'ordonnées de $\mathrm{H}$. Labrouste, qui élimine les variations parasites à plus courte ou plus longue période, donnant finalement une courbe où des maximums apparaissent à intervalles de 17 à 33 mois dont la moyenne est exactement 24,4 mois, avec une amplitude crête à crête de $5,4 \gamma$. La figure 2 présente aussi les séries

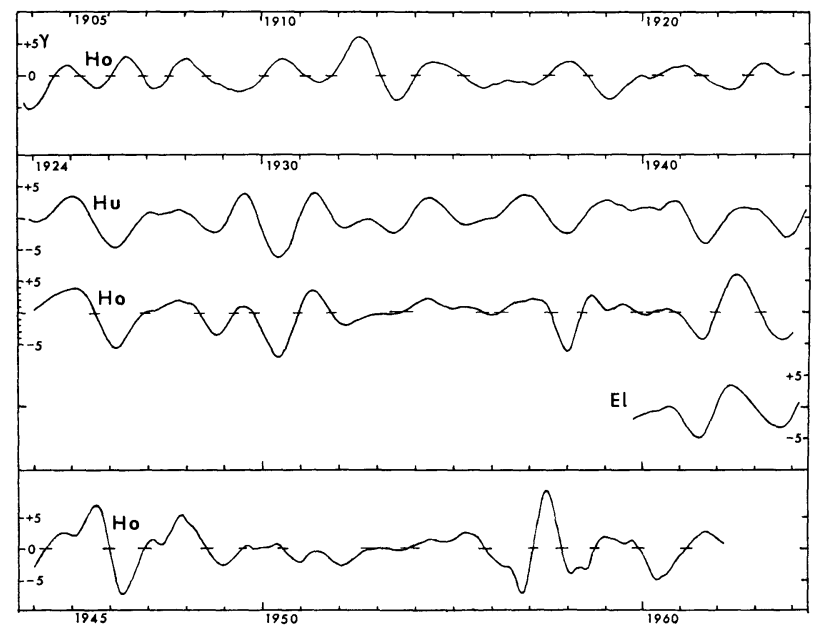

Frg. 2. - Variation biennale de $H$ : de haut en bas : Honolulu (1903-1923), Huancayo (1924-1944), Honolulu (1923-1943), Élisabethville (1939-1944), Honolulu (1944-1963).

d'Élisabethville (1938-1945) où l'on trouve encore des fluctuations de 20 à 26 mois en bonne concordance avec Huancayo, et d'Honolulu, station particulièrement intéressante parce que, située au centre de l'océan Pacifique, on peut considérer que la circulation générale atmosphérique y est à l'état pur, sans les perturbations causées par le relief continental.

Cette station a fourni, pour les années communes, un bon parallélisme avec Huancayo, et, de 1903 à 1925, une tendance biennale aussi nette : de 1905,0 à 1925,0, l'espacement moyen des maximums est de 24,2 mois, mais avec des fluctuations de phase par suite des valeurs dispersées de 18 à 35 mois des intervalles entre les crêtes : seule la méthode de Labrouste permet, dans l'étude d'une variation périodique, de tenir compte de ces changements de phase au cours du temps.

Les données plus récentes d'Honolulu donnent un assez curieux résultat : la variation biennale, effacée avant 1954, reprend une nouvelle vigueur entre 1955 et 1960 , qui sont précisément les années où a été mise en évidence la période de Reed (biennale des vents stratosphériques équatoriaux) [5]. Il sera donc intéressant, dans les années qui viennent, d'examiner la régularité de cette variation.

Sur les résultats calculés jusqu'ici nous remarquerons que l'amplitude de la variation biennale obtenue est de l'ordre du quart de l'amplitude de la variation undécennale. Or l'amplitude biennale des vents stratosphériques zonaux à l'île Canton a été trouvée de $25 \mathrm{~m} / \mathrm{s}$, également le quart de ce que nous avons considéré comme nécessaire pour expliquer au moyen de l'ionosphère la variation undécennale de $H$. Il suffirait donc d'admettre que la variation des vents stratosphériques se répercute jusqu'à l'altitude de l'ionosphère; or une oscillation biennale des mouvements ionosphériques a précisément été mise en évidence par la suite au-dessus de l'Europe centrale [6]. Dans le cas de la variation undécennale, nous envisagions un vent constant dans un milieu dont l'ionisation prend des valeurs variables au cours du cycle solaire, ce qui est un fait d'expérience : dans le cas de la variation biennale, c'est une ionisation constante dans un vent variable qui intervient dans notre discussion.

Nous croyons donc avoir démontré l'existence d'un effet magnétique au sol de la circulation générale. Elle entraîne des conséquences importantes pour l'explication de phénomènes encore incomplètement connus tels que le renversement du champ magnétique à certaines époques du passé géologique.

En effet, le champ $\Delta H=20 \gamma$ que la variation undécennale retranche de la valeur de $H$ correspond à une augmentation de $10^{5}$ ions par $\mathrm{cm}^{3}$ à une altitude $(100 \mathrm{~km})$ où le nombre d'atomes de gaz atmosphérique est supérieur à $10^{13}$. Imaginons que le taux d'ionisation soit multiplié par 1000 : le nombre d'atomes neutres pour un atome ionisé serait encore de l'ordre de $10^{5}$, le libre parcours moyen restant le même; les collisions d'un atome ionisé avec les atomes neutres étant $10^{5}$ fois plus fréquentes que celles des atomes ionisés entre eux, le mécanisme d'entraînement des ions + par le milieu ambiant pourra jouer intégralement : or si $\Delta H$, multiplié lui aussi par 1000 , atteint $20000 \gamma, H$ se trouve annulé au sol, à la suite d'un accroissement d'ionisation de la haute atmosphère hypothétique mais tout à fait plausible dans des conditions de rayonnement solaire accru, et une augmentation supplémentaire de ce taux d'ionisation, d'un facteur 2 seulement, imposerait un champ horizontal de direction inverse et de valeur égale en valeur absolue au champ actuel. Cet effet peut être général sur toute la surface du globe, mais, selon la distribution de la circulation générale de l'atmosphère, il peut être aussi seulement régional : on expliquerait alors les anomalies de la répartition des aimantations inversées de laves volcaniques qui ont été signalées [7].

Supposons maintenant que $H$, au lieu de sa valeur actuelle de 0,5 gauss, ait une valeur beaucoup plus 
petite, par exemple celle $5 \gamma$ du champ interplanétaire. Ce champ suffira encore à créer une couche de transition dans l'atmosphère, mais à une altitude plus élevée, la fréquence de collision des électrons devenant inférieure à la fréquence gyromagnétique au-dessus de $290 \mathrm{~km}$ le jour, $150 \mathrm{~km}$ la nuit.

Les ions positifs constitués par hypothèse d'atomes d'oxygène ionisés sont entraînés dans le mouvement de l'atmosphère tant que leur circonférence gyromagnétique :

$$
C=\frac{2 \pi m v}{\mathscr{H} e}
$$

est supérieure à 4 fois le libre parcours moyen.

Pour $\mathscr{H}=5 \times 10^{-5}$ gauss, on trouve $C=210 \times v \mathrm{~cm}$, où $v$ est la vitesse du vent par rapport au champ magnétique; le libre parcours moyen est donné en fonction de l'altitude par la figure 3 . En conservant $v=10^{4} \mathrm{~cm} / \mathrm{s}$, la couche de transition aura pour limite supérieure $350 \mathrm{~km}$ le jour, $170 \mathrm{~km}$ la nuit.

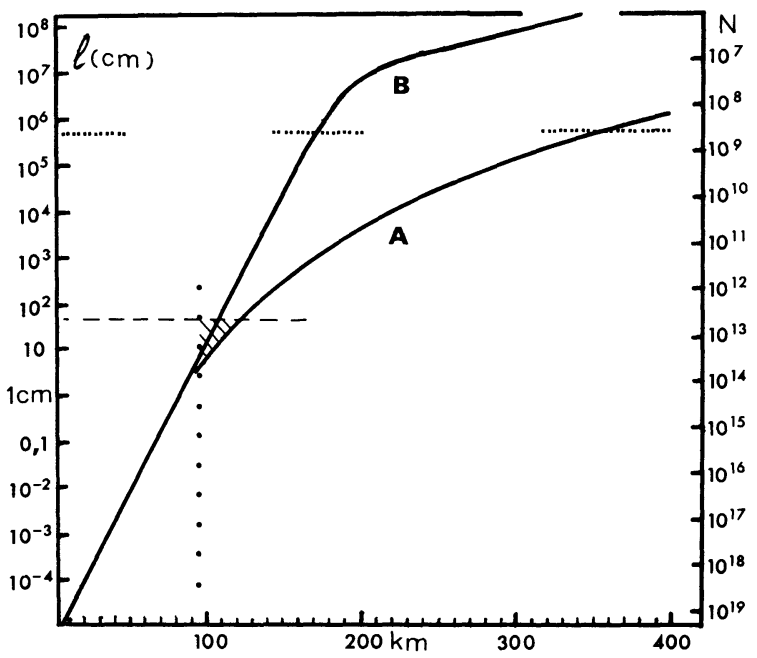

FIG. 3. - Libre parcours moyen $l$ des molécules ou atomes de l'air (échelle de gauche) et nombre $N$ de ces particules par $\mathrm{cm}^{3}$ (échelle de droite) en fonction de l'altitude.

Courbe A : le jour; B : la nuit.

- - - : $1 / 4$ de la circonférence gyromagnétique pour une vitesse de $100 \mathrm{~m} / \mathrm{s}$ et un champ : $\mathscr{H}=0,5$ gauss. ................. : id. pour la même vitesse et pour $\mathscr{H}=0,00005$ gauss.

On a indiqué en hachures la zone de transition où le libre parcours moyen des ions est inférieur au 1/4 de leur circonférence gyromagnétique (pour $H=0,5$ gauss), et la fréquence gyromagnétique des électrons supérieure à leur fréquence de collision.

A ces altitudes où l'ionisation est maximale $\left(10^{6} \mathrm{au}\right.$ lieu de $10^{5}$ par $\mathrm{cm}^{3}$ ), le nombre de particules neutres est encore plus de 100 fois supérieur au nombre de particules ionisées, qui continueront donc à suivre le mouvement du milieu ambiant, et le champ magnétique au sol, toujours calculé par la formule $H=2 \pi i$, aurait une valeur diurne de $600 \gamma$, bien plus grande que le champ initial qui a provoqué la séparation des composantes électriques du milieu atmosphérique : nous nous trouvons donc en présence d'un phénomène de croissance spontanée d'un champ magnétique naturel.

De plus, si le champ de $5 \gamma$ que nous considérons n'est pas lié au globe terrestre, une composante due à la rotation se superpose à la vitesse des couches atmosphériques. Elle est, à l'équateur, de $480 \mathrm{~m} / \mathrm{s}$, ce qui, compte tenu de l'augmentation d'épaisseur de la couche de transition, donne $7500 \gamma$ pour le champ maximal au sol. Dans ce cas, la rotation d'Ouest en Est de la Terre engendrera un champ magnétique du sens actuel.

Il suffirait alors que le taux d'ionisation atmosphérique soit augmenté dans la faible proportion de 1 à 5 pour que le champ magnétique atteigne par ce processus la valeur normale de la composante horizontale du champ terrestre à l'équateur, et le champ ainsi développé sera rendu permanent s'il s'exerce au moment du passage à travers le point de Curie des matériaux de l'écorce terrestre en cours de refroidissement, comme cela s'est produit dans les extrusions successives des dorsales océaniques.

A vrai dire, nous ne disposons pas de données prouvant que la thermorémanence de ces matériaux et surtout leur volume total soient suffisants pour obtenir le moment magnétique de la Terre entière, et nous ne savons pas non plus comment se comporte exactement le champ hypothétique extérieur à la Terre. Mais nous pouvons considérer que la formation d'un champ magnétique est une conséquence inévitable de la nature physique de notre atmosphère.

Il est intéressant de remarquer que l'absence de champ magnétique sur les corps célestes les plus voisins de la Terre prend place aisément dans le cadre de notre théorie, la Lune étant dépourvue d'atmosphère, Mars n'en ayant qu'une très ténue où le taux d'ionisation est probablement très faible, et l'ionosphère de Vénus étant elle aussi inférieure à celle de la Terre, sans doute en raison de sa composition différente, et la rotation de cette planète étant très lente, tandis que l'ionisation des hautes couches de l'atmosphère de Jupiter, du même ordre de grandeur que la nôtre, est multipliée par une vitesse de rotation 27 fois plus grande.

On peut même se demander si les champs mesurés dans le Soleil et les étoiles n'ont pas leur origine dans un mécanisme semblable puisque le champ obtenu ne dépend que du taux d'ionisation des gaz en mouvement et de leur vitesse. Comme celle-ci dépend de la rotation de l'astre, nous comprendrions ainsi qu'on ait remarqué une relation entre le moment magnétique et le moment de rotation de certains corps célestes [8].

\section{BIBLIOGRAPHIE}

[1] Bernard (P.), Ann. Géophys., 1952, 8, 2, 248.

[2] Bernard (P.), C. R. Acad. Sci., 1952, 234, 866.

[3] GEORGE (E. F.), Proc. Inst. Radio-Engin., 1947, 35, 3, 249.

[4] Bernard (P.), C. R. Acad. Sci., 1966, 263 B, 317.

[5] REED (R. D.) et Rogers (D. G.), J. Atm. Sc., 1962, $19,127$.
[6] SPRenger (K.) et Schminder (R.), Z. Meteorol., 1967, 19, 5-6, 168.

[7] Bonhommet (N.) et Babkine (J.), C. R. Acad. Sci., 1967, 264 B, 92.

[8] Blacketrt (P. M. S.), Nature, 1947, 159, 658. 